\title{
Paired, Total, and Connected Domination on the Queen's Graph Revisited
}

\author{
Paul A. Burchett \\ 1005 Riverside Ave, Kingsport, USA \\ Email: burchettd4@gmail.com
}

Received 18 August 2015; accepted 14 December 2015; published 17 December 2015

Copyright (C) 2016 by author and Scientific Research Publishing Inc.

This work is licensed under the Creative Commons Attribution International License (CC BY). http://creativecommons.org/licenses/by/4.0/

(c) $\underset{\mathrm{EY}}{\mathrm{E}}$ Open Access

\section{Abstract}

The question associated with total domination on the queen's graph has a long and rich history, first having been posed by Ahrens in 1910 [1]. The question is this: What is the minimum number of queens needed so that every square of an $n \times n$ board is attacked? Beginning in 2005 with Amirabadi, Burchett, and Hedetniemi [2] [3], work on this problem, and two other related problems, has seen progress. Bounds have been given for the values of all three domination parameters on the queen's graph. In this paper, formations of queens are given that provide new bounds for the values of total, paired, and connected domination on the queen's graph, denoted $\gamma_{t}\left(Q_{n}\right), \gamma_{p r}\left(Q_{n}\right)$, and $\gamma_{c}\left(Q_{n}\right)$ respectively. For any $n \times n$ board size, the new bound of $\gamma_{c}\left(Q_{n}\right) \leq\left\lceil\frac{2 n}{3}\right\rceil$ is arrived at, along with the separate bounds of $\gamma_{t}\left(Q_{n}\right) \leq\left\lceil\frac{2 n}{3}\right\rceil-1$, for $n \equiv 3,4,5(\bmod 6)$ with $n \geq 21$, and $\gamma_{p r}\left(Q_{n}\right) \leq\left\lceil\frac{2 n}{3}\right\rceil-1$, for $n \geq 22$ with $n \equiv 4(\bmod 6)$.

\section{Keywords}

Chess, Total Dominating Set, Paired Dominating Set, Connected Dominating Set

\section{Introduction}

Interest in domination type problems began with the introduction of the Five Queen's problem by De Jaenisch in 1862 [4]. Stated simply the Five Queen's problem is this: What is the minimum number of queens needed to attack or occupy every square of a standard $8 \times 8$ chessboard? This problem has been extended in many different ways by mathematicians and chess enthusiasts to include generalizations of this problem to other pieces, board 
sizes, board types, domination parameters, etc. For a good survey on the extensions of this and other chessrelated mathematics problems see [5].

It often helps when dealing with these types of problems to use the tools of graph theory. For example, the extension of the original problem to include any $n \times n$ board can be described by associating squares with vertices. Then, we form the $n \times n$ Queen's graph, denoted $Q_{n}$, by adding an edge between any two of our $n^{2}$ vertices whose corresponding squares share a common row, column, or diagonal. Any two vertices are said to be adjacent if they have an edge between them. The other extensions of De Jaenish's original problem that will be dealt with in this paper are the considerations of three types of domination parameters on the $n \times n$ queen's graph, namely those of total, paired, and connected domination.

Along these lines, a set of queens is said to be a total dominating set if and only if every square on our $n \times n$ board is attacked. A set of queens is said to be a paired dominating set if and only if they attack every square of our $n \times n$ board, and there exists a perfect matching among the vertices corresponding to the squares occupied by queens. By perfect matching we mean that the queens can be grouped into disjoint sets of adjacent pairs, with all queens accounted for in exactly one of the sets. In this way, a paired dominating set of queens can be placed on the board, two at a time, in attacking pairs.

Lastly, a set of queens is a connected dominating set if and only if every square on our board is attacked or occupied, and given any two vertices corresponding to occupied squares there exists a path between these vertices consisting solely of vertices associated with occupied squares. In other words, one can move between any two vertices in the set using any number of movements among vertices in our set, but only by moving from vertex to vertex where adjacency exists-making sure to repeat no vertex. The minimum values among all total, paired, or connected dominating sets on the queen's graph are denoted by $\gamma_{t}\left(Q_{n}\right), \gamma_{p r}\left(Q_{n}\right)$, and $\gamma_{c}\left(Q_{n}\right)$ respectively. For more terminology related to these domination parameters see [6].

\section{Results}

Theorem 1. For all $n,\left\lceil\frac{2 n}{3}\right\rceil-1 \leq \gamma_{c}\left(Q_{n}\right) \leq\left\lceil\frac{2 n}{3}\right\rceil$.

Proof: It should be noted that the lower bound of $\left\lceil\frac{2 n}{3}\right\rceil-1 \leq \gamma_{c}\left(Q_{n}\right)$ was arrived at in [3]. Also, for $n<12$, it was shown in [2] [3] that the upper bound holds. Next, we will show $\gamma_{c}\left(Q_{n}\right) \leq\left\lceil\frac{2 n}{3}\right\rceil$ for $n \geq 12$.

Suppose $n \equiv 0(\bmod 6)$ with $n \geq 12$. Divide the board into nine regions, as in Welch's formation of queens seen in [3]. Label the regions from left to right, bottom to top, beginning with region one in the bottom left corner of our $n \times n$ board. Beginning with the bottom-right corner of the subboard formed by region five, place queens in every square to the right of the vertical axis of symmetry in the bottom row of the $\frac{n}{3} \times \frac{n}{3}$ subboard, every square in the left-most column of region five below the horizontal axis of symmetry, every square in the top-most row of region five to the left of the vertical axis of symmetry, and finally, every square in the rightmost column of region five above the horizontal axis of symmetry. Note our total count on the number of queens is $\frac{2 n}{3}$. Figure 1 will illustrate.

To see that the queens form a connected set of queens, consider any two vertices corresponding to queens in the set. One can then denote a path between them where the vertices in our path correspond to a clockwise ordering of queens in our formation, beginning with either of the two considered vertices.

Next, to see that they are indeed a dominating set, consider regions two, four, five, six, and eight. It is easy to see that these regions are dominated either row-wise, column-wise, or both by the queens in region five.

Now consider the symmetric cases of regions one, three, seven, and nine. It is easy to see that these regions' $\frac{2 n}{3}-1$ diagonals (sum diagonals in the case of regions one and nine, difference diagonals in the case of regions three and seven) are all dominated by the $\frac{2 n}{3}$ queens with the only overlap among the diagonals being a sum 


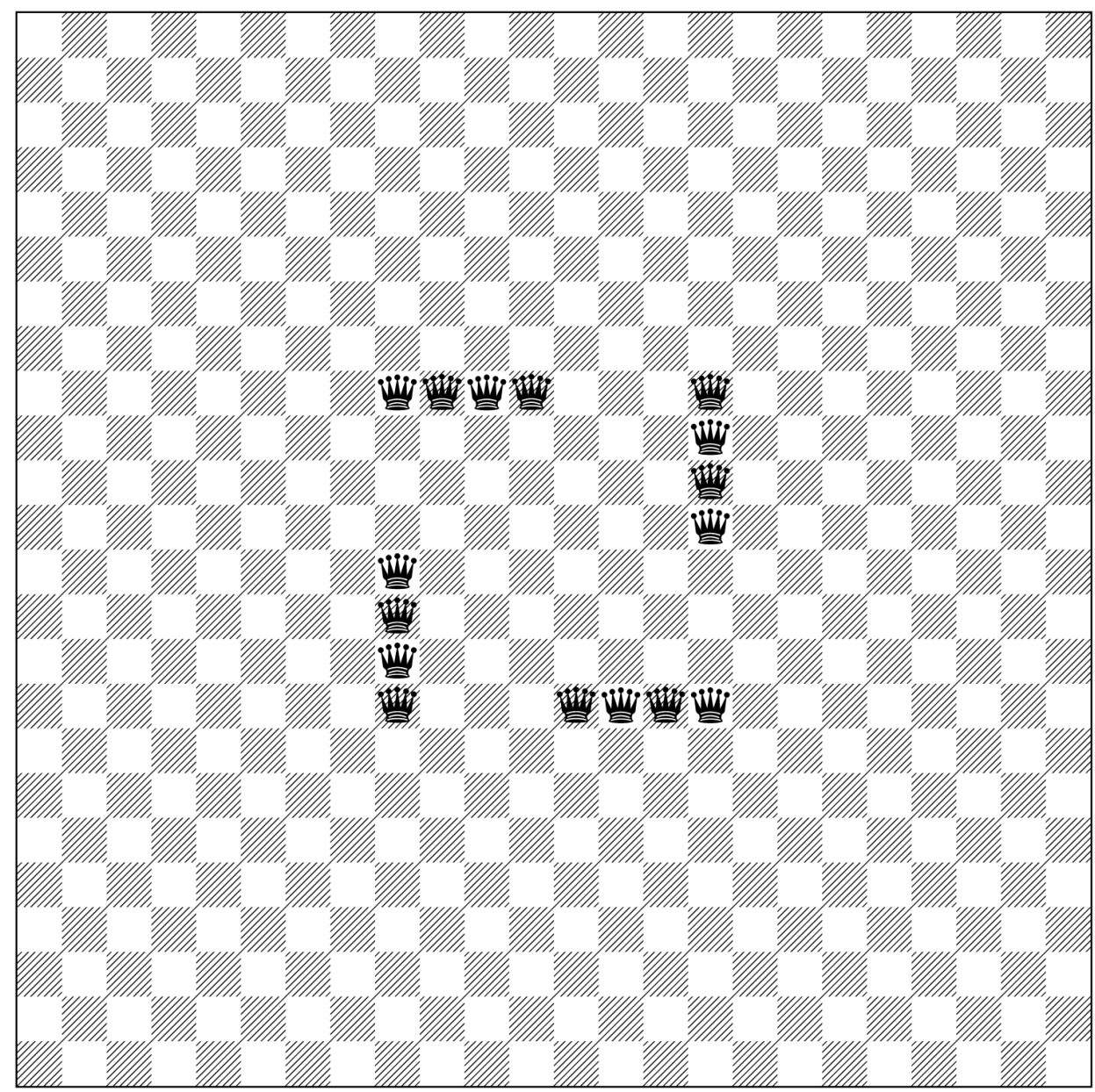

Figure 1. A formation of 16 connected and dominating set of queens on a $24 \times 24$ board.

and difference diagonal edge associated with the four queens along the two main diagonals.

For the case of $n \equiv 1(\bmod 6)$, form an $(n-1) \times(n-1)$ subboard in one of the corners of our $n \times n$ board and place queens as in Figure 1. Then place an additional queen at the intersection of the added row and column. It is easy to see this is a connected dominating set of $\left\lceil\frac{2 n}{3}\right\rceil$ queens since the original formation was connected, and the added queen occupies one of the main diagonals along with exactly two other queens in the set.

In the case where $n \equiv 2(\bmod 6)$, form an $(n-2) \times(n-2)$ subboard in one of the corners of the board and place queens similar to what is seen in Figure 1. Then, place queens along the main diagonal at the intersection of the added rows and columns. Likewise, it is easy to see this forms a connected dominating set of $\left\lceil\frac{2 n}{3}\right\rceil$ queens.

Now consider the case for $n \equiv 3(\bmod 6)$ with $n \geq 15$. Again, as in case for which $n \equiv 0(\bmod 6)$, divide the board into nine regions and label them in a similar manner as above. We will begin by placing queens in a similar way as in Figure 1, with those to the right of the vertical line of symmetry and on the bottom-most row in the border squares of region five having queens placed in them, and the others placed along the border of region five, with the same rules as above, save for the corner squares of region five and those on the axes of symmetry. The corner squares of region five do not have queens placed in them. Thus, the total count for the number of queens thus far is $\frac{2 n}{3}-6$.

Next, place queens in the center of the left-most and right-most columns of region five and a queen in the 
center of the board. To cover the diagonals adjacent to the corner squares of region five we place queens on the squares whose center has coordinates $\left(0,-\frac{n}{3}+1\right)$ and $\left(0, \frac{n}{3}-1\right)$, with the origin taken to be the center of the center square. Then lastly, to “connect” the three components, place a queen at $\left(-1, \frac{n}{3}-1\right)$, since this square is adjacent to queens from all three components. This brings our count to exactly $\frac{2 n}{3}$ queens. Figure 2 will illustrate.

It is easy to see that, in a similar manner as for the case of $n \equiv 0(\bmod 6)$, regions two, four, five, six, and eight are dominated either row-wise, column-wise, or both by the formation of queens, with or without the final queen placed. Thus, all that is left to consider are the four corner regions of the board-each of which has exactly $\frac{2 n}{3}-1$ positive and negative diagonals. It is then easy to see that, for any of the regions noted, there is a 1-1 correspondence between the diagonals under consideration in these regions and the queens in the formation before the final queen is placed.

To see the cases where $n \equiv 5(\bmod 6)$ form an $(n-2) \times(n-2)$ subboard placed in one of the corners of our $n \times n$ board. Place queens as for the case where $n \equiv 3(\bmod 6)$ in our $(n-2) \times(n-2)$ subboard, then place queens at the intersection of the added rows and columns along one of the main diagonals. It is easy to see this is a connected dominating set of queens.

It should be noted that an upper bound of $\left\lceil\frac{2 n}{3}\right\rceil$ for $\gamma_{c}\left(Q_{n}\right)$, with $n \equiv 4(\bmod 6)$, was previously arrived at in [7]. This, along with the above theorem, reduces $\gamma_{c}\left(Q_{n}\right)$-values to, at most, two possibilities for any size $n$

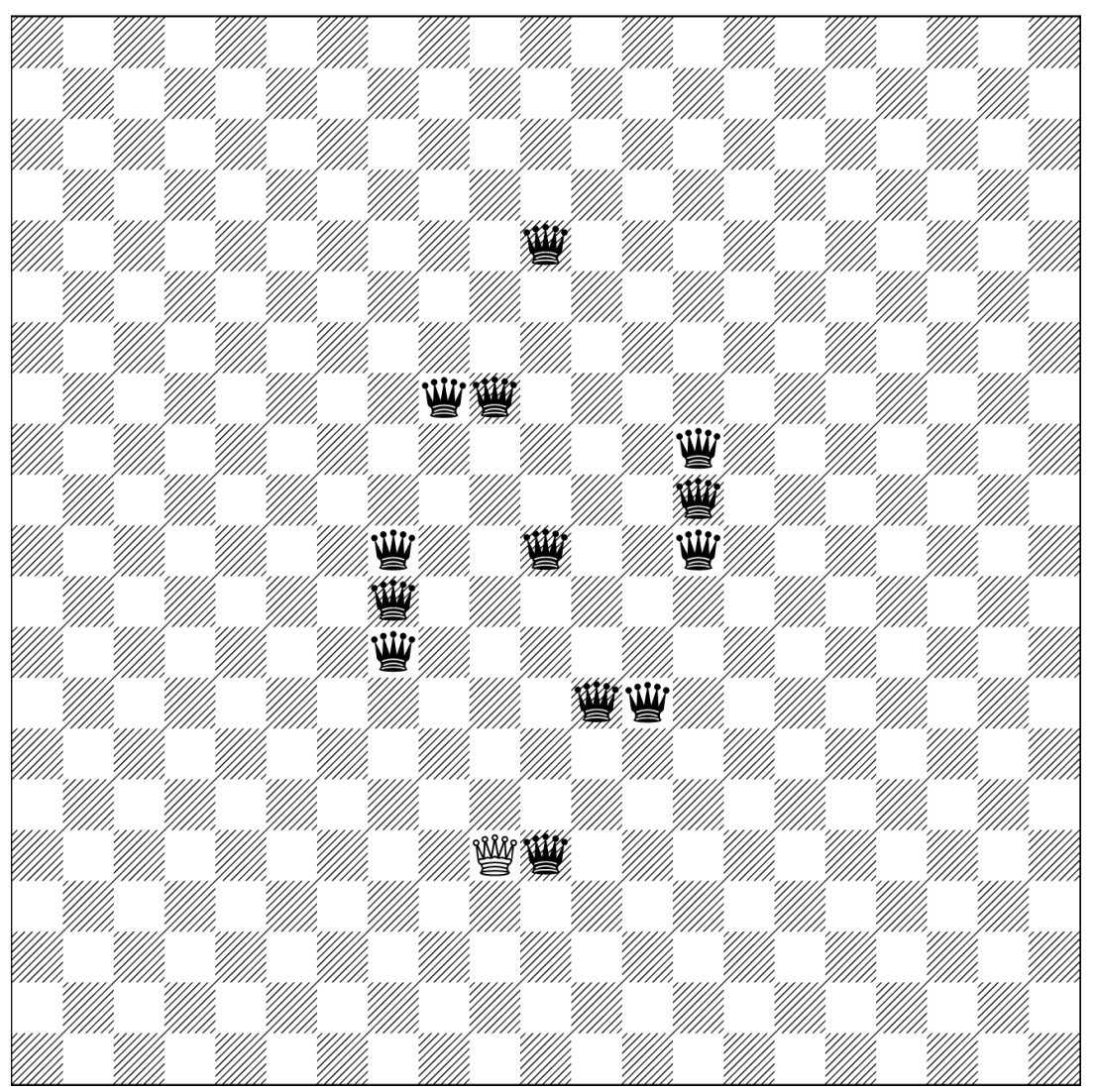

Figure 2. A formation of 14 connected and dominating set of queens on a $21 \times 21$ board. The queen placed last is in white. 
with $\gamma_{c}\left(Q_{n}\right)=\left\lceil\frac{2 n}{3}\right\rceil-1$ known to hold for $n=6$ and $\gamma_{c}\left(Q_{n}\right)=\left\lceil\frac{2 n}{3}\right\rceil$ for $2 \leq n \leq 5, \quad 7 \leq n \leq 11$, and $n=13,14,16,17,19,20,22,23$.

Lemma 1. For $n \equiv 3,4,5(\bmod 6)$ and $n \geq 21, \gamma_{t}\left(Q_{n}\right) \leq\left\lceil\frac{2 n}{3}\right\rceil-1$.

Proof: The proof for the upper bound is similar to Theorem 1 in that we use the same formation as in Figure 2, save for the final queen placed. There will be three components in the subgraph associated with our queens, each of which for $n \geq 21$ has cardinality of greater than two-thus supplying us with a total dominating set of $\frac{2 n}{3}-1$ queens.

For the case where $n \equiv 4(\bmod 6)$ place queens as in Figure 2 in any $(n-1) \times(n-1)$ subboard, save for the final queen. Then place a queen in the intersection of the added row and column. It is easy to see this forms a total dominating set of $\left\lceil\frac{2 n}{3}\right\rceil-1$ queens since the added queen is diagonally adjacent to the queen in the center of our $(n-1) \times(n-1)$ subboard.

For the case where $n \equiv 5(\bmod 6)$, place queens as in Figure 2 in any $(n-2) \times(n-2)$ subboard, save for the final queen. Then, place queens in the intersection of the added rows and columns along one of the appropriate main diagonals.

Lemma 2. For $n \geq 22$ and $n \equiv 4(\bmod 6), \gamma_{p r}\left(Q_{n}\right) \leq\left\lceil\frac{2 n}{3}\right\rceil-1$.

Proof: To see the proof use the same formation of total dominating queens as for the case of $n \equiv 4(\bmod 6)$ as in Lemma 1. The perfect matching can be arrived at by matching the queen at the center of the $(n-1) \times(n-1)$ subboard with the queen at the intersection of the added row and column. Then, match the two queens that are both column-adjacent to the center queen. The two queens row-adjacent to this center-most queen are likewise matched. This leaves us with two columns with an even number of queens, and two rows with an even number of queens. These can be matched in a straight forward manner.

\section{Summary}

It should be noted that the lower bound of $\left\lceil\frac{4(n-1)}{7}\right\rceil \leq \gamma_{t}\left(Q_{n}\right) \leq \gamma_{p r}\left(Q_{n}\right)$ was arrived at in [3] for all $n$. This lower bound matches the known value for $\gamma_{t}\left(Q_{n}\right)$ in the cases where $n \leq 10$, or $n=12,15,17,18,19$ and $\gamma_{p r}\left(Q_{n}\right)$ when $2 \leq n \leq 10$, or $n=12,13,15,16,17,18,19,20,23$ [3]. For the cases where $n \leq 21, \gamma_{t}\left(Q_{n}\right)$ values have been shown to either attain this lower bound or have a value that is, at most, one greater than the mentioned lower bound. Likewise, given any $n \leq 21, \gamma_{p r}\left(Q_{n}\right)$-values have been reduced to, at most, two possible values. This leads us to the following conjecture.

Conjecture 3. $\lim _{n \rightarrow \infty} \frac{\gamma_{t}\left(Q_{n}\right)}{n}=\lim _{n \rightarrow \infty} \frac{\gamma_{p r}\left(Q_{n}\right)}{n}=\frac{4}{7}$.

For larger $n$, with $n \equiv 0,1,2(\bmod 6)$, the current best upper bound for $\gamma_{t}\left(Q_{n}\right)$ is still the bound of $\gamma_{t}\left(Q_{n}\right) \leq\left\lceil\frac{2 n}{3}\right\rceil$ provided by Welch's formation. Likewise, for $n \neq 4(\bmod 6)$ and larger $n$, the current best upper bound is provided by Welch's formation of queens, or a slight manipulation of this formation for paired domination. Better upper bounds than those provided by either Welch's formation, or those provided above for both $\gamma_{p r}\left(Q_{n}\right)$ and $\gamma_{t}\left(Q_{n}\right)$ for large $n$ seem desirable.

\section{References}

[1] Ahrens, W. (1910) Mathematische unterhaltungen und spiele. B.G. Teubner, Leipzig-Berlin.

[2] Amirabadi, A. (2005) Values of Total Domination Numbers and Connected Domination Numbers in the Queen's Graph. Master's Thesis, Clemson University, Clemson. 
[3] Burchett, P.A. (2006) Paired, Total and Connected Domination on the Queen's Graph. Congressus Numerantium, 178, 207-222.

[4] De Jaenisch, C.F. (1862) Applications de l’Analyse Mathematique au Jeu des Echecs. Petrograd.

[5] Watkins, J.J. (2004) Across the Board: The Mathematics of Chessboard Problems. Princeton University Press, Princeton and Oxford. http://dx.doi.org/10.1515/9781400840922

[6] Haynes, T.W., Hedetniemi, S.T. and Slater, P.J., Eds. (1998) Domination in Graphs: Advanced Topics. Marcel Dekker, New York.

[7] Burchett, P.A. (2011) On the Border Queens Problem and k-Tuple Domination on the Rook’s Graph. Congressus Numerantium, 209, 179-187. 